\title{
PERÍODO DE TRANSICIÓN DEL NIÑO Y EL ADOLESCENTE A LA ADULTEZ TRATADO CON HORMONA DE CRECIMIENTO RECOMBINANTE (RHGH). UNA REALIDAD
}

\author{
Transition period of child and adolescent to \\ Adults treated with Growth Hormone \\ Recombinant (RHGH). A reality
}

\section{Francisco Carvajal Martíneza , Yuraimi Piz Ramos ${ }^{\mathrm{b}}$ y Mónica Carvajal Aballe}

Recibido: 3 de marzo, $2019 \bullet$ Aprobado: 6 de junio, 2019

Cómo citar: Carvajal Martínez F, Piz Ramos Y, Carvajal Aballe M. Período de transición del niño y el adolescente a la adultez tratado con Hormona de Crecimiento Recombinante (RHGH). Una realidad. cysa [Internet]. 17 de junio de 2020 [citado 17 de junio de 2020];4(2):13-20. Disponible en: https://revistas.intec.edu.do/index.php/cisa/article/view/1767

\section{Resumen}

La baja talla por Deficiencia de Hormona de Crecimiento es una de las causas endocrinas más frecuentes. El tratamiento se realiza con hormona de crecimiento recombinante (rhgh) durante un período habitualmente de larga duración. Existe un grupo de pacientes que su tratamiento con hormona de crecimiento no concluye en la adultez y debe ser tratado también en ese periodo de la vida. El tema de las transferencias se planteó por primera vez en Estados Unidos en 1984, como consecuencia del importante aumento de supervivencia durante los años setenta y ochenta de niños con patología crónica y discapacitante. Se entiende por transición a una etapa de cambios físicos y psicológicos que, de forma arbitraria, se extiende desde el final de la pubertad hasta que la maduración adulta se completa. Es preciso reevaluar los pacientes con deficiencia de hormona de crecimiento tras alcanzar la talla adulta y definir el estado de la secreción de GH para valorar la conti el periodo de transición y en la adultez.

Palabras clave: período de transición; hipopituitarismo; deficiencia de hormona de crecimiento.

\footnotetext{
a Profesor Titular y Consultante de Endocrinología. Investigador Titular y de Mérito. Especialista de Primer y Segundo Grado en Endocrinología. Departamento de Endocrinología Pediátrica. Instituto Nacional de Endocrinología. La Habana, Cuba. Correo-e: endoped@infomed.sld.cu
}

\begin{abstract}
The sort stature due to growth hormone deficiency is one of the most frequent endocrine cause. The treatment is carried out with recombinant growth hormone (rhgh) for a period of usually long duration. There is a group of patients treatment with growth hormone does not end in adulthood and should also be treated at that period of life. The issue of transfers we first raises in United States in 1984, as a consequence of the significant increase in survival during 1970-1080 in children with chronic and disabling diseases. The transition period from child to adult represents a crucial phase in the growth process where multiple physical and psychosocial changes occur. It has been arbitrarily defined as the period extending from late puberty to full adult maturity. It is necessary to reassessment patient with Growth Hormone Deficiency after that to arrive the adult height and to define the $\mathrm{GH}$ secretion status for to evaluate the continuity of replace treatment during the transition and adult period.
\end{abstract}

Keywords: Transition period; Growth Hormone Deficiency; hypopituitarism; short stature.

\footnotetext{
b Especialista Primer Grado en Endocrinología. Instituto Nacional de Endocrinología. La Habana, Cuba. Orcid: 0000-0002-4577-4486

${ }^{\mathrm{c}}$ Especialista de Primer Grado en Oncología Médica, Instituto Nacional de Oncología, La Habana, Cuba. Orcid: 0000-0002-1453-0526
} 


\section{Introducción}

El déficit de hormona de crecimiento (DGH) es una afección de naturaleza congénita o adquirida, se caracteriza por la ausencia parcial o total de la GH detectable en el plasma.

El DGH es la forma más común de hipopituitarismo, siendo más frecuente en niños que en niñas (2:1). La incidencia varía significativamente entre diferentes países y se estima que sea alrededor de 1:4000-1:10000 individuos.

Es conocido que el tratamiento con hormona de crecimiento $(\mathrm{GH})$ comenzó en 1958 por Raben, y durante una gran etapa ha sido siempre orientado a niños y adolescentes con deficiencia de esta hormona.

A lo largo de los ańos se ha ido ganando en experiencia en la sustitución hormonal en este eje (GH/ IGF-1), transitándose por una serie de etapas: ${ }^{1}$

- 1945-1970: los nińos con problemas endocrinos (incluidos los desórdenes del crecimiento) eran referidos a los endocrinólogos de adultos o a pediatras generales, pues el perfil pediátrico dentro de la endocrinología aún no estaba desarrollado.

- 1970-1985: el endocrinólogo pediatra se incorpora al tratamiento de la Deficiencia de Hormona de Crecimiento (DGH).

- 1985-2000: se amplían las indicaciones del tratamiento con hormona de crecimiento, extendiéndose su uso hasta el adulto con DGH.

- Posterior al 2000: esta última etapa ha comenzado, y se caracteriza por la colaboración estrecha de los endocrinólogos (pediátricos y de adultos) en el tratamiento con análogos de la GH (rhGH) ganando más experiencia en este campo.
El primer objetivo de la terapia de reemplazo en nińos con deficiencia de hormona de crecimiento (DGH) es normalizar la línea de crecimiento. ${ }^{2}$ Otras metas de este tratamiento son mejorar la composición corporal, la densidad mineral ósea, la calidad de vida y el perfil metabólico; así como reducir el riesgo de enfermedad cardiovascular. ${ }^{2}$

\section{Periodo de transición}

El tema de las transferencias se planteó por primera vez en Estados Unidos en 1984, como consecuencia del importante aumento de supervivencia durante los años setenta y ochenta de nińos con patología crónica y discapacitante. ${ }^{3}$

En la última década, se ha insistido en la importancia del denominado período de transición. La transición ha sido definida con el propósito de planear la transferencia de los adolescentes y adultos jóvenes con una condición física y clínica crónica, de un centro pediátrico a uno orientado a la atención de adultos. $^{4}$

Por periodo de transición se entiende una etapa de cambios físicos y psicológicos que, de forma arbitraria, se extiende desde el final de la pubertad hasta que la maduración adulta se completa. En relación con la etapa del crecimiento comprende, habitualmente, los 6 a 7 años posteriores al momento en el que el niño adquiere la talla adulta ${ }^{5}$ y desde el punto de vista práctico es el período entre los 15-25 años de edad.

Es conocido que el desarrollo del cerebro no se completa hasta que termina la maduración sexual en la pubertad. En esta fase se desarrollan las relaciones psicosociales y se forma la identidad personal, ${ }^{6}$ por lo que la transición ocurre al mismo tiempo que otras fases de la adaptación social, como el abandono del hogar, los retos vocacionales y convivir con una pareja; fenómenos que complejizan aún más este periodo. 
La transición requiere de cuidados con la contribución del endocrinólogo pediátrico y de adulto. ${ }^{5,7,8} \mathrm{El}$ momento de la transferencia del servicio pediátrico hacia el de adultos debe ser planeado desde edades tempranas, en consenso individualizado con cada paciente, sin establecer una edad específica. ${ }^{6}$ Es imprescindible, la orientación adecuada en estos pacientes y sus familiares para que no abandonen la atención médica al cambiar el centro de atención y el endocrinólogo de asistencia. El proceso puede ser estructurado, incluyendo la educación del paciente y los familiares sobre los efectos del tratamiento y la autodeterminación en el manejo de su salud. Según Dowing et al., ${ }^{9}$ más de la cuarta parte de los pacientes con enfermedad endocrina crónica no acuden regularmente a las consultas de seguimiento, posterior a la transición.

\section{Cambios relacionados con el crecimiento físico, que ocurren durante la transición}

Durante esta etapa van concluyendo algunos procesos que se inician desde la niñez y van progresando paulatinamente hacia la adultez, existiendo gran variabilidad individual y especificidades de cada sexo: ${ }^{10}$

- El crecimiento longitudinal se considera terminado cuando la velocidad de crecimiento es menor a 1,5-2,5 cm/año.

- La maduración ósea es del 97-98\%.

- El pico de masa ósea se alcanza entre los 20 $\mathrm{y}$ los 25 ańos.

- La masa muscular aumenta hasta los 20 años en los varones y hasta los 14 años en las mujeres.

- La masa grasa aumenta hasta los 20 años en las mujeres y hasta el final de la pubertad en los hombres.

La maduración corporal, puede demorar su desarrollo total hasta casi los 30 años ya que el desarrollo somático no se ha completado al finalizar el creci- miento y los estudios disponibles muestran evidencias que indican que la acción de la hormona de crecimiento es necesaria en la fase de transición posterior a la pubertad para alcanzar el estatus normal del adulto. ${ }^{11}$

\section{Evaluación}

El principal objetivo de la terapia con rhGH en niños con DGH es normalizar el crecimiento durante la infancia y obtener la talla adulta esperada según su meta genética. ${ }^{12,13}$

A pesar de los avances en el campo, este tratamiento sustitutivo sigue siendo controvertido. Por un lado, la importancia del mantenimiento de una calidad de vida adecuada; por el otro, el riesgo de aumentar la morbi-mortalidad del paciente a largo plazo. Se impone el reto de conocer los beneficios y perjuicios del mantenimiento o suspensión de esta terapia, por lo que resulta importante determinar:

1) La evolución de la maduración tisular en los adolescentes.

2) Las consecuencias de la interrupción del tratamiento.

3) El efecto de la sustitución con GH en la morbilidad por fracturas y la enfermedad cardiovascular.

Existen diferentes aspectos importantes que se valoran en el manejo del período de transición del paciente con DGH: ${ }^{12}$

1. Reevaluación de la etiología y manejo de la enfermedad específica.

2. Reevaluación del régimen terapéutico con $\mathrm{GH}$ con vista a similar la producción disminuida de la secreción endógena de esta hormona.

3. Alcanzar el total desarrollo somático del adulto, incluyendo la masa corporal y la densidad mineral ósea. 
4. Completar la maduración puberal, sexual y reproductiva.

5. Reducción de los riesgos metabólicos y cardiovasculares.

6. Lograr el desarrollo psicosocial adulto.

7. Educación para que los pacientes tengan conocimiento de su enfermedad en orden de desarrollar autonomía en sus decisiones sobre el cuidado de su salud.

Hasta épocas recientes, el tratamiento con GH se suspendía cuando se alcanzaba la talla adulta final, sin embargo, en la actualidad, se valoran nuevos conceptos y se acepta que el desarrollo muscular y óseo no se completa alcanzando valores de adultos hasta etapas posteriores (alrededor de los 25 años de edad) por lo que se ha planteado la posibilidad de continuar el tratamiento en la adultez.

Se impone la necesidad de reevaluar (en conjunto, el endocrinólogo pediátrico y el de adulto, encargado de su seguimiento futuro) en la etapa de transición, al final del periodo de crecimiento longitudinal, la capacidad de secreción de GH en todos los casos de déficit de $\mathrm{GH}$, excepto aquellos que recibieron tratamiento con rhGH, pero no son realmente deficitarios; por ejemplo, las niñas con Síndrome de Turner. ${ }^{11,14-17}$

El tratamiento debe ser interrumpido cuando el desarrollo puberal y el crecimiento se haya completado, el intervalo entre la reevaluación y la suspensión del tratamiento no debe ser menor de un mes ni mayor de 3 meses $^{5}$ y el resto de los déficits hormonales deben estar corregidos, considerando el efecto de los estrógenos sobre los niveles de IGF-1. Los niveles de GH vuelven a su estado basal alrededor de una semana tras la supresión del tratamiento, pero el IGF-1, IGFBP-3 y la subunidad ácido lábil (ALS) pueden tardar entre 6 y 12 meses. ${ }^{14,16}$

El "gold standard" para reevaluar el DGH es el test de hipoglucemia inducida por insulina, sin embargo, no existen diferencias en cuanto a sensibilidad y especificidad entre este y el test de ARG-GHRH. ${ }^{18}$

Si los resultados durante la reevaluación de: IGF-1 y de los test de liberación de GH (preferiblemente la hipoglucemia inducida por insulina y/o la ARG$\mathrm{GHRH}^{15}$ son bajos, el diagnóstico de deficiencia de GH está confirmado y se deberá valorar mantener el tratamiento con rhGH, lo cual es frecuente es la deficiencia de hormona de crecimiento severa y múltiple, no así en la deficiencia idiopática y selectiva de hormona de crecimiento. Al evaluar los niveles de GH debemos valorar la variabilidad según el IMC. Así, se considera déficit de GH: ${ }^{10}$

- $\mathrm{IMC}<25 \mathrm{~kg} / \mathrm{m} 2, \mathrm{GH}<11 \mu \mathrm{g} / \mathrm{l}$;

- IMC $25-30 \mathrm{~kg} / \mathrm{m} 2, \mathrm{GH}<8 \mu \mathrm{g} / \mathrm{l}$,

- $\mathrm{IMC}>40 \mathrm{~kg} / \mathrm{m} 2, \mathrm{GH}<4 \mu \mathrm{g} / \mathrm{l}$.

Respecto al punto de corte de GH, es importante recordar que la secreción de $\mathrm{GH}$ no es la misma a los 16, por ejemplo, que a los 50 años y, por ello, no es lógico utilizar siempre el mismo valor. La propuesta de La Sociedad Europea de Endocrinología Pediátrica (ESPE) es utilizar siempre y para todos los estímulos un punto de corte de $5 \mu \mathrm{g} / \mathrm{l}$. Un valor de GH inferior a 5,1 $\mu \mathrm{g} / \mathrm{l}$ en el test de hipoglucemia inducida por insulina, equivale a un valor menor de 4,15 $\mu \mathrm{g} / \mathrm{l}$ con GHRH + arginina (95\% de sensibilidad y $92 \%$ de especificidad para el déficit de GH del adulto). ${ }^{19}$

Si se utilizan los criterios de la ESPE en la respuesta a los test de estímulo de $\mathrm{GH}$, podemos establecer tres categorías de diagnóstico según la edad en que sea valorado el eje GH/IGF-1:12

- Déficit de GH en la infancia: pico de GH inferior a $10 \mu \mathrm{g} / \mathrm{l}$.

- Déficit de GH en la transición: pico de GH inferior a $5 \mu \mathrm{g} / \mathrm{l}$.

- Déficit de GH en el adulto: pico de GH inferior a $3 \mu \mathrm{g} / \mathrm{l}$. 


\section{Tratamiento}

En la práctica existen varios grupos de pacientes para valorar la continuidad del tratamiento: ${ }^{10}$

1. Aquellos con deficiencia severa (pico de $\mathrm{GH}<3$ $\mu \mathrm{g} / \mathrm{l})$ según el test de estimulación con insulina $\mathrm{u}$ otro alternativo.

2. Los que presentan deficiencias múltiples hipofisarias (aproximadamente el 91-100\% de este grupo persisten con deficiencia de $\mathrm{GH}$ en la adultez). Por el contrario, solo el 20-30\% de los que tienen deficiencia de GH idiopática parcial tienen déficit severo.

3. Pacientes que no han alcanzado el pico de la masa ósea.

4. Un nuevo grupo que serían los transicionales supervivientes de cáncer y que han recibido radioterapia.

En los pacientes sin alcanzar el pico de masa ósea, ya sea con inicio en la niñez o en la adolescencia, se podría administrar tratamiento con rhGH hasta los 25 años de edad. En estas situaciones las dosis de rhGH serían más altas que en la adultez, aproximadamente 2,0 mg/día, otros señalan dosis de 0,2-0,5 $\mathrm{mg} /$ día. ${ }^{10}$

Tras alcanzar la talla final no se debe usar la dosis pediátrica de $25 \mu \mathrm{g} / \mathrm{kg} /$ día, sino utilizar la de aproximación a la del adulto, manteniendo la IGF-1 normal (entre $0 \mathrm{y}+2 \mathrm{DE}$ ), comenzando con 0,2 a $0,5 \mathrm{mg} /$ día. Es necesario que la dosis de rhgh sea de 3-6 veces mayor que la utilizada habitualmente en el adulto. En la transición, rara vez se precisan más de $2 \mathrm{mg}$ diarios. En las recomendaciones de la ESPE se aconseja iniciar con $0,2 \mathrm{mg} /$ día en varones y $0,3 \mathrm{mg} /$ día en mujeres. ${ }^{12}$

La dosis de la terapia de reemplazo es individual, los adultos jóvenes pueden beneficiarse con dosis más elevadas: 400-500 $\mu \mathrm{g} /$ día. La dosis pudiera ser aumentada en 100-200 $\mu \mathrm{g} /$ día cada uno o dos meses hasta obtener una buena respuesta clínica y valores de IGF-1 dentro del rango de referencia según la edad y sin efectos colaterales.

Nos pudiéramos preguntar ¿por qué sería necesario el tratamiento con rhGH en estos pacientes que persiste la deficiencia de la hormona de crecimiento después de la reevaluación?

Es conocido que los pacientes con deficiencia de $\mathrm{GH}$ en la adultez pueden presentar numerosas afecciones, dentro de ellas tenemos:

Fallo en la calidad de vida: depresión y disminución de la satisfacción de vivir, irritabilidad, disminución de la disposición a alcanzar metas, pobre imagen corporal y de socialización, disminución de la energía, poca concentración y disminución de la memoria y fatiga mental.

Cambios en la composición corporal: disminución de la masa muscular, incremento de la masa grasa, especialmente del tronco (grasa visceral), deshidratación y disminución del sodio corporal, disminución de la tolerancia al ejercicio, hipertermia y disminución de la sudoración durante la actividad física, incremento del 7-8 \% de la masa grasa que la correspondiente a la edad, sexo y talla.

Maduración en general: los pacientes con inicio en la niñez de la deficiencia de hormona de crecimiento y que son tratados solamente hasta la talla final sufren una deficiencia significativa de maduración, la masa corporal, esquelética, muscular y, probablemente, grasa adquieren un desarrollo sub-óptimo, ya que existe un fallo relativo del anabolismo

Metabolismo óseo: presentan disminución de la masa ósea, disminución de la densidad mineral, disminución del turnover óseo, retraso en la mineralización, ya que existen bajos niveles de marcadores de la formación ósea: osteocalcina y procolágeno 
e incremento del riesgo de fractura ósea. Las alteraciones de la densidad mineral ósea pueden estar relacionadas con: inicio de la deficiencia de $\mathrm{GH}$, sexo y deficiencia de otros ejes hipofisarios.

Metabolismo de carbohidratos: insensibilidad relativa a la insulina, fallo de la tolerancia a la glucosa.

Alteraciones lipídicas: incremento de la masa grasa, incremento de los triglicéridos, niveles elevados de colesterol total y de LDL-colesterol, concentraciones de HDL-colesterol baja, los cambios lipídicos correlacionan con la severidad de la deficiencia de hormona de crecimiento, principalmente en la mujer, al parecer el trastorno lipídico ocasionaría o facilitaría la aterosclerosis prematura y la enfermedad cardiovascular.

Trastornos hematológicos: anormalidades de factores de la coagulación que sugieren un defecto del sistema fibrinolítico, aumento del fibrinógeno, elevación a nivel tisular del plasminógeno activador del inhibidor -1 (PAI-1), concentraciones elevadas del factor VII, niveles elevados de citoquinas pro inflamatorias (interleucina-6 y factor alfa de necrosis tumoral).

Manifestaciones cardiovasculares: incremento de la mortalidad cardiovascular, disminución del "output" cardíaco, estructura cardíaca anormal (disminución de la masa ventricular izquierda), inadecuada fracción de eyección, incremento de la presión arterial sistólica, aumento de la homocisteína y correlación entre el funcionamiento cardíaco con la severidad de la deficiencia de hormona de crecimiento.

Relación con la aterogénesis: se ha encontrado placas ateroscleróticas en arterias femorales y carotídeas, se ha observado incremento del engrosamiento de la íntima-media de las arterias carotídeas, al parecer existe disfunción endotelial e incremento del estrés oxidativo.
Mortalidad/morbilidad: existe incremento de la mortalidad en el paciente hipopituitario sin tratamiento con $\mathrm{GH}$, más marcado en la mujer por infarto del miocardio y eventos cerebrovasculares. Se observa el incremento de 3.8 veces más el riesgo relativo de mortalidad.

Otro aspecto importante a evaluar son los costos de salud: existe incremento del costo médico-social para el paciente hipopituitario por no empleo, retiro laboral temprano, enfermedad por depresión y necesidad de una pensión. Se ha encontrado que el $11 \%$ de los hombres y el $31 \%$ de las mujeres con hipopituitarismo requieren ayuda diaria para sus actividades cotidianas.

Además del costo general, existe el costo específico, que incluye el factor de riesgo cardiovascular y el posible incremento de fracturas. Se ha estimado que en estos pacientes el costo de salud es $35 \%$ más alto que el promedio nacional.

Los beneficios de la continuidad del tratamiento se exponen a continuación:

\section{Efectos de la sustitución de GH en el período de transición ${ }^{7,16,19}$}

\section{Composición corporal}

- Aumento de masa muscular.

- Disminución de masa grasa.

- Incremento de masa ósea.

- Las mujeres ganan una pequeña cantidad de masa muscular sin cambios en la masa grasa.

- Los hombres ganan bastante masa muscular y pierden una cantidad significativa de masa grasa.

- El cambio en la relación masa ósea/talla es mayor en los hombres. 


\section{En el hueso}

- En déficit de GH al inicio de la infancia hay una reducción significativa del grosor cortical y de la densidad mineral ósea.

- La suspensión de la GH en la transición limita la adquisición del pico de masa ósea y promueve el desarrollo de osteopenia en la edad adulta.

- El tratamiento con GH aumenta el contenido mineral óseo en los casos de déficit más grave de GH.

\section{Metabólicos}

- El déficit de GH en la transición produce aumento de colesterol total, colesterol LDL y apoproteína B con disminución de HDL. Con la sustitución, los cambios son variables, según los autores: sin cambios en LDL, colesterol HDL y TG, o bien aumento de HDL o disminución de LDL.

- El tratamiento con GH disminuye la sensibilidad a la insulina (clamp euglucémico) a pesar de la mejoría simultánea de la composición corporal.

\section{Calidad de vida}

- El único dato con alguna capacidad predictiva es la calidad de vida basal. El sexo, la edad, la intensidad del déficit, otros déficits hormonales asociados, los cambios de composición corporal o los niveles de IGF-1 no tienen ningún valor predictivo.

Es por eso que opinamos, como posible conclusión, que los médicos que tratamos estos pacientes en las edades pediátricas debemos considerar en algunos niños, la continuación del tratamiento con rhGH en el adolescente con deficiencia de hormona de crecimiento que transita a la adultez. Debe estar basada en una cuidadosa valoración posterior al finalizar el tratamiento, que incluya la reevaluación de la persistencia del déficit de GH después de haber alcanzado la talla final, así como la orientación adecuada al endocrinólogo de adulto que continuará con su seguimiento. La información de todas las decisiones tomadas, debe ser obligatoriamente brindada a la familia y al paciente, ya que es fundamental su apoyo en esta importante etapa del tratamiento.

\section{Bibliografía}

1. Witt JM, Rekers LT, Tuers GB, Drowe B, Beck TJ, et al. Growth hormone treatment to finally height in children with idiopathic short stature: Evidence for a dose effects. J Pediatr. 2005; 146:45-53

2. Wilson TA, Rose SR, Cohen P, et al. Update of guidelines for the use of growth hormone in children: The LawsonWilkins Pediatric Endocrinology Society Drug and Therapeutics Committee, J. Pediatr. 2003;143:415-21.

3. Blum RW. Introduction. Pediatricas. 2002; 110:1301-3.

4. Blum RW, Garell D, Hodgman $\mathrm{CH}$, et al. Transition from child centred to adult health-care systems for adolescents with chronic conditions. A position paper of the Society of Adolescent Medicine. J Adolesc Health 1993; 14:570-6.

5. Argente J, Álvarez-Escolá C, de Miguel-Novoa P. Tratamiento con hormona de crecimiento en el período de transición del niño al adulto. Rev Horm Crecim. 2011;16:105-6.

6. Kapellen TM, Kiess, W. Transition of adolescents and Young adults with endocrine diseases to adult health care. Best Prac Res Clin Endocrinol Metab.2015; 29(3):505-13. 
7. Clayton P, Gleeson H, Monson J, et al. Growth hormone replacement throughout life: Insights into age-related responses to treatment. Growth Horm IGF Res. 2007;17:369-82.

8. Savage MO, Drake WM, Carroll PV, et al. Transitional care of GH deficiency: When to stop GH therapy. Eur J Endocrinol. 2004; 151:S61-65.

9. Downing J, Gleeson HK, Clayton PE, et al. Transition in endocrinology: the challenge of maintaining continuity. Clin Endocrinol 2013; 78:29-35.

10. Álvarez CE, Fernández J. M, Recio et al. Documento de consenso del área de conocimiento de Neuroendocrinología de la Sociedad Española de Endocrinología y Nutrición para el abordaje del hipopituitarismo durante la transición. Endocrinol Nutr. 2014;61(2):1-11.

11. Motlich ME, Clemmons DR, Malowzowski S, et al. Evaluation and treatment of adult growth hormone deficiency: an Endocrine Society Clinical practice guideline. J Clin Endocrinol Metab 2012; 96:1587-609.

12. Clayton PE, Shalet SM. Difficulties in patient management associated with the transition period. Guidelines of themanagement of the young GHD adult. Horm Res 2005;64(Suppl. 2):74-5.

13. Sawicki GS, Kelemen S, Weitzmann ER. Ready, set, stop: mismatch between self-care beliefs, transition readiness skills, and transition planning among adolescents, young adults and parents. Clin Pediatr. 2014; 53:1062.
14. Radovick S, DiVall S. Approach to the growth hormone-deficient child during transition to adulthood. J Clin Endocrinol Metab. 2007; 92:1195-2000.

15. Geffner ME. Transition to the adult endocrine clinic; testing pituitary function what test and when? Growth Horm IGF Res. 2003; 13:117-21.

16. Maghnie M, Strigazzi C, Tinelli C, et al. Growth hormone (GH) deficiency (GHD) of childhood onset: Reassessment of GH status and evaluation of the predictive criteria for permanent GHD in young adults. J Clin Endocrinol Metab. 1999; 84:1324-8.

17. Inzaghi E, Cianfarani S. The challenges of growth hormone deficiency diagnosis and treatment during the transition from puberty to adulthood. Front Endocrinol. 2013; 4:1-8.

18. Dörr HG, Hauffa BP, Wallaschowski H, for the national KIGS and KIMS Boards. Management of adolescents with childhood onset growth hormone deficiency in the transition e results of a field based study in Germany. Dtsch Med Wochenschr 2009; 134:2551-5.

19. Leong GM, Johannsson G. Growth hormone deficiency: Strategies and indications to continue growth hormone therapy in transition from adolescence to adult life. Horm Res. 2003; 60:78-85. 